
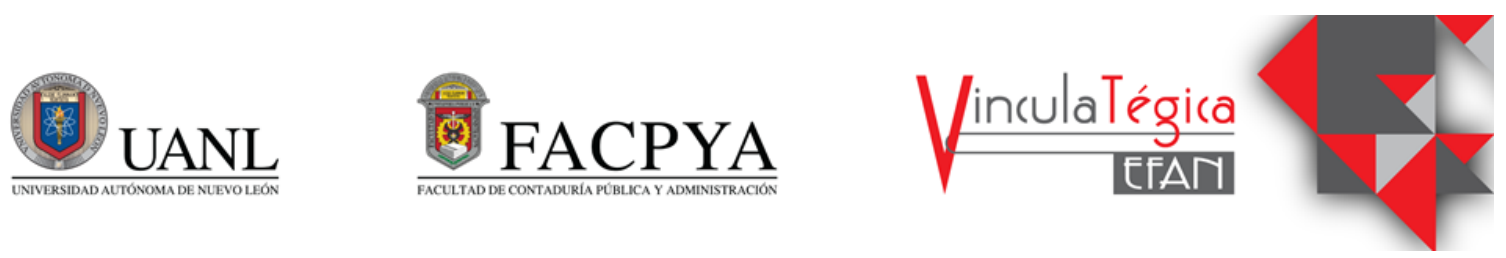

\title{
ELEMENTOS QUE MEJORAN LA COMPETITIVIDAD DE LAS EMPRESAS MEDIANAS INDUSTRIALES EN NUEVO LEÓN
}

\author{
Luis Enrique Lechuga Calderón ${ }^{1}$, Mónica Blanco Jiménez ${ }^{2}$ y Patricio Galindo Mora ${ }^{3}$ \\ ${ }^{1}$ Universidad Autónoma de Nuevo León-FACPYA, luislechigacld.@uanl.edu.mx, Ave. Universidad s/n, Ciudad \\ Universitaria, San Nicolás de los Garza, N.L., Tel: 83294080 \\ ${ }^{2}$ Universidad Autónoma de Nuevo León-FACPYA, monica.blancojm.@uanl.edu.mx, Ave. Universidad s/n, Ciudad \\ Universitaria, San Nicolás de los Garza, N.L., Tel: 83294080 \\ ${ }^{3}$ Universidad Autónoma de Nuevo León-FACPYA, patricio.galindom.@uanl.edu.mx, Ave. Universidad s/n, Ciudad \\ Universitaria, San Nicolás de los Garza, N.L., Tel: 83294080
}

\begin{abstract}
Información del artículo revisado por pares
Fecha de aceptación: junio-2021

Fecha de publicación en línea: diciembre-2021

DOI: https://doi.org/10.29105/vtga7.2-49
\end{abstract}

\section{Resumen}

En general, a nivel mundial, las pequeñas y medianas empresas aportan una base sólida a las economías nacionales ya que dan ocupación, empleo y actividad en proporciones mucho mayores de la población que las grandes empresas, por eso su participación en el PIB mexicano es mayor tanto en ingresos, ganancias y representación. Si bien los países siempre buscan la mejora continua y el desarrollo y crecimiento de las compañías, siempre existen grandes brechas en materia de productividad y digitalización entre las PYMES y las grandes empresas. Para que las PYMES tengan procesos eficientes y eficaces que permita darles una mayor competitividad se requieren impulsar algunos elementos. Por eso el objetivo de esta investigación fue determinar los elementos que inciden en la competitividad de las PYMEs industriales localizadas en el Estado de Nuevo León. Después de una revisión teórica exhaustiva se propuso que estos factores son: la utilización de recursos tecnológicos, la innovación en productos y procesos, tener finanzas sanas, contar con un plan de negocios, capacitar al personal y finalmente tener sistemas de control como variables independientes. La metodología utilizada fue la elaboración de un instrumento de 35 preguntas que midieran la correlación de estas variables usando la estadistcia de regersion lineal. Los resultados muestran que aunque hay dos variables significativas:

INNOVACIÓN y CAPACITACIÓN, el marco teórico permite dar sustento a las otras variables que apoyan la competitividad. Por lo que es importante destacar que otras empresas pueden utilizar esta metodología que permita medir estas variables en sus empresas. 


\begin{abstract}
Worldwide, small and medium enterprises represent a solid base for local economies since they move the economy and give job to people in a greater volume than big companies. Meanwhile countries are always looking for development, improvement on the economy and seek to create opportunities, there have always been big gaps between SME's and big companies. Currently, the weight that SME's have in the local economies in terms of income, earnings, GDP, etc is similar to the weight that big enterprises have but the fact that there are millions of small and medium companies and just a few big enterprises makes it not equilibrated because a few companies make big income and millions of SME's makes little. Due this situation, this document tries to propose a methodology or a guideline that SME's could follow to use and measure some variables that could improve competitivity. These variables are the usage of technological resources, Innovation in product and processes, maintain a scenario with Healthy Finances, have and follow a Business Plan, Train the personnel and have a good Control system. It was concluded that these factors well applied were key to become a more competitive company. Although, every company will have to identify what factor has to be ranked top priority because every market will present different obstacles and they must be sorted accordingly to the specific case.
\end{abstract}

\title{
Key words
}

Competitiveness, Industry, Innovation, Technology, Training.

\section{INTRODUCCIÓN}

Las PYMES son un elemento indispensable en la transformación económica de los países ya que representan una fuerza indispensable en el desarrollo económico sobre todo porque forman parte de la cadena de valor de grandes empresas (SAP Blogs, 2013). Como muestra están las estadísticas del Banco Mundial que indican que las PYMES contribuyen hasta en un 45\% del empleo formal total y en un 33\% del ingreso nacional (PIB) en las economías emergentes. Sobre todo, numerosos estudios han demostrado que las PYMES contribuyen al crecimiento del empleo, aún más que las tasas altas de las grandes corporaciones, lo que representa una importante porción del empleo total. Además, en las economías emergentes, el rol de las pequeñas y medianas empresas es fundamental para la promoción de la actividad emprendedora, el fomento de la innovación y la capacidad de diversificación. De hecho, de acuerdo con Durán, Paloma (2017), las micro, pequeñas y medianas compañías suponen aproximadamente el 90\% del sector empresarial en todo el mundo y no solo eso, sino que su papel es particularmente relevante en los países en desarrollo, ya que actúan como auténticos motores de la creación de empleo y el crecimiento económico.

Respecto a la situación de las PYMES en México, la relación entre la ocupación y empleos de PYMES es similar. Esto lo reafirma Neuman (2020) cuando menciona que la Pequeña y Mediana Empresa (PYME) en México tiene un gran potencial de desarrollo, ya que según las más recientes cifras que genera la Secretaría de Economía existen cerca de 4’000,000 de empresas catalogadas como PYMES, lo que representa el 99\% del total 
de las empresas que operan en nuestro país, y que además dan empleo al 72\% de la población económicamente activa, generando aproximadamente el 52\% de participación del PIB nacional. Como lo señalan Canal, Lukas (n.d.), las PYMES tienen, en promedio, una productividad tres veces menor en relación con las grandes empresas y, por ende, ofrecen salarios 50\% más bajos a sus empleados. Lo anterior, se sustenta con datos del Grupo del Banco Mundial, que confirman que las empresas más grandes son más productivas y tienen mayor facilidad para adquirir e implementar nuevas tecnologías.

En el ámbito específico de las PYMES en Nuevo León, según la DENUE (2021), hasta el 2020 existían más de 5,000,000 de entidades económicas catalogadas como PYMES, de las cuales, aproximadamente 186,500 se localizan en Nuevo León, un 3.7\% del total. Si bien, podría parecer muy poco, esto no es proporcional a la aportación que el estado realiza al PIB nacional. Nuevo León aporta aprox. un 7.6\% del PIB nacional de acuerdo con información oficial del gobierno mexicano, siendo las actividades secundarias las más significativas y que dan un mayor aporte (Económica, 2019). Por lo que esta PYMES representan aproximadamente el 97\% del volumen de las empresas y aportan el 50\% del PIB en Nuevo León.

Tomando esto en cuenta, esta investigación se focaliza en el siguiente objetivo que es determinar los elementos que permiten mejorar la competitividad de las empresas medianas industriales en Nuevo León. Por lo que para contestar a la siguiente pregunta de investigación ¿Cuáles son esos elementos?, se propone como hipótesis que contar con recursos tecnológicos, innovación en productos, tener finanzas sanas, contar con un plan de negocios, capacitación y tener sistemas adecuados de control son los elementos que permiten a las empresas medianas industriales ser más competitivas en el mercado local.

Es necesario realizar la investigación porque va a traer beneficios a todos los involucrados como lo son las empresas ya que define los factores necesarios para tener mayor competitividad. A los dueños o gerentes porque les proporcionará un panorama de lo que se requiere lograr para ser una empresa competitiva, a los empleados para que entienden las estrategias que se requiere implementar, también a los emprendedores ya que este estudio les dará una base para ajustar sus planes y corregir las estrategias que planeen y a los estudiantes porque les ayudará a ver todo lo que hace a una empresa competitiva y comprenderán más fácilmente lo visto en clase.

Para dar sustento a las variables dependiente e independientes propuestas se presentará a continuación el sustento teórico que fundamentarán estas y eso permitirá validar y contrastar los resultados obtenidos y sobre todo hacer epistemología aplicada y teórica del conocimiento existente sobre cada una de las variables.

\section{MARCO TEÓRICO}

\subsection{Variable dependiente "Competitividad"}

\section{- Teorías y definiciones de la competitividad}


La competitividad es definida por la Real Academia Española como la capacidad que se tiene de competir con el rival en la búsqueda de un fin. Por su parte Labaca (2007) señala la definición de competitividad de Porter como "la capacidad de una empresa para producir y mercadear productos en mejores condiciones de precio, calidad y oportunidad que sus rivales" (p. 160). Según estos textos, se entiende que el concepto "competitividad" podría definirse como la capacidad de ser más efectivo, rápido y productivo que las entidades que representan rivalidad en el mercado. Ser competitivo se traduce en ofrecer mejores servicios con calidad y buenos precios.

Para evaluar la influencia de un país sobre la competitividad internacional de sus empresas existe el modelo de Michael Porter que propone que: la demanda, las industrias relacionadas, la estrategia y rivalidad de las empresas, son los determinantes de la competitividad de una industria específica (Labaca, 2007). Porter menciona en su modelo que las ventajas más valiosas son aquellas que requieren gran esfuerzo o capital para desarrollar o aplicar la competitividad. Respecto a las condiciones de la demanda, se menciona que tendrá gran influencia en la industria ya que la demanda local podría forzar a las industrias a convertirse en economías de escala o bien, a incursionar en los mercados extranjeros en una etapa temprana. Respecto a las industrias relacionadas se menciona que habrá una mayor ventaja si se trabaja y se tiene mayor relación con proveedores y empresas del grupo que tengan los mismos objetivos y enfoque con tecnología. Muy similar a lo que se habla en el factor de estrategia, estructura y rivalidad en donde habrá más competitividad si se cuentan con industrias alineadas en esta estrategia, tanto regional como nacionalmente según comenta Labaca (2007).

Figura 1 Modelo Porter

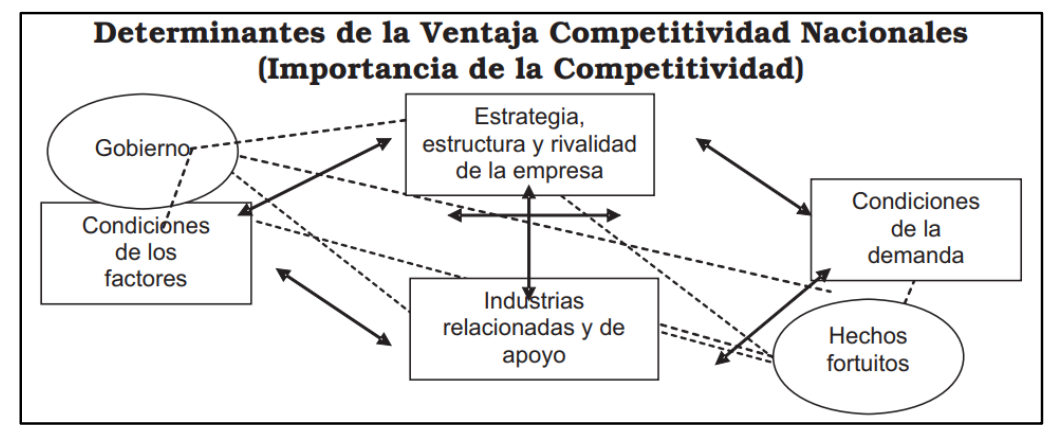

Fuente: Labaca (2007)

\section{- Estudios aplicados sobre la competitividad}

La búsqueda de la competitividad tiene varios años, como los señala la investigación de Singh, Garg y Deshmukh (2008) que revisaron más de 130 documentos internacionales acerca de las estrategias de desarrollo de las PYMES para ser más competitivas. Los rubros identificados como posibles áreas de mejora para aumentar competitividad son tales como: el Apoyo de dirección y gerencia al equipo, la cultura organizacional, la administración total de la calidad, el desarrollo de alianzas con proveedores, la administración de la cadena de suministros, la administración de la innovación y conocimiento, la investigación y desarrollo de productos y las tecnologías de la información. 
Otro elemento clave para mejorar la competitividad en las PYMES mexicanas es la innovación, como lo señala González y De La Parra (2017) que si se quiere ser un país más competitivo, entonces: es hora de innovar, para lo cual se requiere contar con los siguientes elementos: la generación de conocimiento con metodologías, modelos, técnicas o herramientas, la innovación y las nuevas tecnologías. De igual manera, las investigaciones de Ahmedova (2015) a empresas pequeñas y medianas localizadas en Bulgaria y la Unión Europea, señalan que deberían darse apoyos de manera más contundente a las empresas que tienen el potencial de ser competitivas considerando 5 factores que son: acceso al financiamiento, innovación, propiedad intelectual, internacionalización e implementación de la mejora continua.

Ycaza y Sánchez-Riofrío (2016) realizaron una extensa revisión de literatura acerca de los factores que impulsan o fomentan la competitividad basándose en tres criterios. Primeramente, en que la información se encontrase en bases de datos reconocidas a nivel mundial, segundo que fueran artículos recientes y tercero, por su relevancia dentro del tema estudiado medido a través del número de citas recibidas. Todos los documentos revisados analizaban a compañías PYMES de America latina ya que se buscaba encontrar información relevante acerca de esta región específica del planeta.

Se evaluaron las siguientes variables como las más importantes para lograr una mayor competitividad en las PYMES; la innovación, la productividad, la calidad, el desarrollo del capital humano, la tecnología e inversión en Investigación y Desarrollo, y finalmente, la gestión financiera y, de acuerdo a los resultados de la investigación se concluye que la competitividad es una condición que toma cada vez mayor relevancia ya que las exigencias del entorno económico actual aumentan y si las empresas se desenvuelven en un entorno favorable teniendo un capital humano propicio para satisfacer necesidades específicas, contando con una excelente infraestructura económica y optando por la innovación como eje en sus bienes o servicios, se logrará ser competitivos.

Además, Ibarra Cisneros, González Torres, Demuner Flores (2017), utilizando una metodología de carácter descriptivo, correlacional y de corte transversal; desarrollaron un modelo de competitividad sistémica a escala micro y aplicaron un instrumento de medición de 64 preguntas a 195 empresas PYMES del giro manufacturero en el estado de Baja California. Además los resultados mostrados de su investigación indican que las PYMES manufactureras de Baja California son medianamente competitivas, pero con tendencia importante hacia la baja, independientemente de su tamaño.

Las variables analizadas son la planeación estratégica, producción y operaciones, aseguramiento de la calidad, comercialización, contabilidad y finanzas, recursos humanos, gestión ambiental y sistemas de información. Los resultados descriptivos muestran que las áreas de contabilidad y finanzas se consideran menos al momento de hacer funcionar una empresa y no existe la visión por parte de los empresarios de utilizarla como punta de partida para lograr que la competitividad de la empresa se incremente. Asimismo, la dimensión que más contribuye a explicar la competitividad de la empresa es producción y operaciones. 
Una última investigación que se aborda respecto a los factores independientes y su relación con la competitividad es la realizada por García y Sánchez (n.d.). Ellos involucran las siguientes 12 variables para evaluar a aproximadamente 394 PYMES ubicadas en el Edo. de Mexico: competencia externa, regulación gubernamental excesiva, mercado deprimido, relaciones laborales, niveles de capacitación de la mano de obra, necesidad de modernización, acceso a capital o recursos financieros, costos de los insumos, costos internos, calidad, ciclos de tiempo para la producción y atención y satisfacción al cliente.

Finalmente un estudio más reciente de Jiménez, Campos \& Vargas-Hernández (2020) incluye como las variables y/o factores internos de las PYMES del sector del comercio en Michoacán que mejoran la competitividad son las que planean estratégicamente, que cuentan con recursos humanos capacitados y experiencia lo cual les permite tomar mejores decisiones, entendiendo la innovación como un proceso permanente donde se privilegie la iniciativa, las nuevas ideas, dónde se rompa con las estructuras establecidas por su parte la calidad es uno de los factores más apreciados para la competitividad y la certificación un medio para el aseguramiento de la misma” (P.17).

A continuación, se mostrará la justificación teórica y/o aplicada de cada una de las variables independientes propuestas y que además de los mencionado anteriormente, le darán una valides de ser utilizadas en esta investigación.

\subsection{Recursos tecnológicos (X1)}

Prasanna, et al. (2019) realizaron una investigación tomando como base cientos de documentos relacionados a las PYMES para determinar las variables relacionadas con los recursos tecnológicos y como favorecen la competitividad de las empresas. Concluyeron en que existen 2 condiciones cruciales para obtener el proceso tecnológico, por una parte, los impulsores y por la otra las condiciones de desarrollo. Esto permitirá tener como resultado una mejor competitividad en los rubros que se presentan en la figura 5.

Figura 2 Teoría de recursos tecnológicos

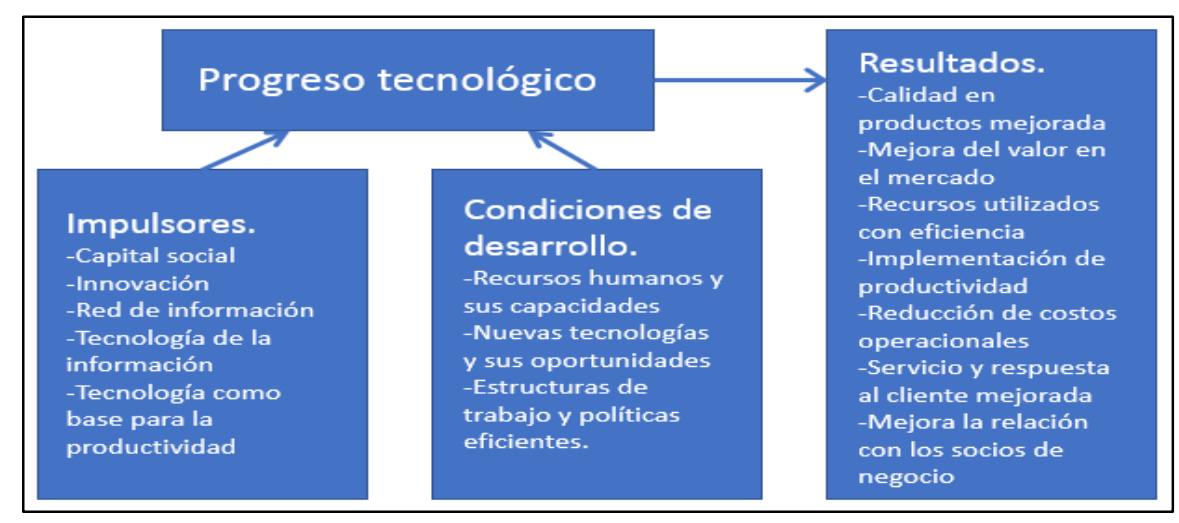

Fuente: Prasanna et al. (2019) 
Entonces, de manera más general y en base a la recopilación de esto conceptos, se puede concluir que los recursos tecnológicos tales como: equipos de cómputo, plataformas de información, big data, softwares CRM, uso de redes sociales, son utilizados por la empresa para apoyarse en mejorar sus actividades, procesos, objetivos, desarrollos, y demás datos. Siempre utilizando la tecnología disponible y las mejores prácticas para hacer eficientes los procesos.

\subsection{Innovación en productos y procesos (X2)}

Al concepto "Innovación" se le da el significado de producir o introducir algo nuevo, alguna idea, algún método o instrumento nuevos para ponerlo en marcha. Por lo que la innovación es la concepción de una idea, la cual requiere de un proceso para que se lleve a cabo en un uso productivo. Es una mejora, actualización o incremento a una práctica, producto o proceso actual. Pinto y Rodríguez-Moreno (2018) señalan que existen 5 tipos de innovación posibles; introducción de nuevos bienes o de bienes de nueva calidad, introducción de un nuevo método productivo (no deriva de algún descubrimiento científico), apertura de un nuevo mercado, conquista de nuevas fuentes de oferta de materias primas, establecimiento de una nueva organización en una determinada industria.

Cuevas (2016) realizó un estudio para comprobar si la innovación y la tecnología tenían inferencia en la competitividad de las PYMES manufactureras en Aguascalientes. Los resultados muestran que la innovación, la tecnología y el capital intelectual infieren positiva y directamente en la competitividad de las PYMES. En pocas palabras, estarse innovando e incluir esta innovación a un esquema tecnológico va a ayudar a que se tengan mejores oportunidades de ser más competitivo.

\subsection{Finanzas sanas / Planeación financiera (X3)}

Para los autores Masilo y Aguirre (2017) la planeación financiera puede definirse como el proceso en donde se describen las actividades económicas y la asignación de recursos de una empresa. Mencionan en su artículo que la falta de planeación empresarial, incluida la planeación financiera, es una de las causas del fracaso de los emprendimientos empresariales.

Este tema es aún más complicado para las PYMES ya que se topan con obstáculos (Saavedra-García et al. de 2016): como no saber manejar o controlar los datos financieros, no tener una planeación de las finanzas, no tener herramientas de inclusión financiera o simplemente no tener un ambiente de libertad financiera en el mercado en el que se desenvuelven (Pavón, 2016). Por ende, se puedo concluir que la planificación financiera ayuda a tener un buen desempeño y control de las finanzas considerando la lógica de que las finanzas sanas ayudarán al manejo financiero y por ende la competitividad de la compañía aumentará.

\subsection{Contar con un plan de negocios (X4)}

Valverde Arana, G. (2011) se refiere a un plan de negocios como fruto de un ejercicio de organización que examina cuantitativa y cualitativamente los precios y beneficios de llevar a cabo una iniciativa de negocios u 
posibilidad de inversión, con el propósito de contribuir a tomar una elección de inversión. Debería enfatizar, además, los aspectos fuertes y ser realista sobre los puntos de vista débiles. Ahora bien, podemos ver que, en el modelo para la elaboración de un plan de negocios de Vargas Urzola, A. (2002) llamado "Business Model Canvas (BMC)" se describen los 9 módulos que identifican la lógica de la empresa para conseguir ingresos ya que estos cuben las cuatro áreas principales de un negocio: clientes, oferta, infraestructura y viabilidad económica. por lo que se debe de incluir un plan de negocio los siguientes datos; aliados clave, actividades clave, recursos clave, propuesta de valor, relación con clientes, canales, segmento de clientes, estructura de costes, flujos de ingresos.

\subsection{Capacitación de personal (X5)}

Rengifo Maco (2018) realizó estudios sobre la capacitación, encontrando algunas definiciones como la de Dessler que señala que la capacitación se basa en otorgar las habilidades necesarias para el desarrollo de su puesto, a través de un programa de inducción. Por lo que, la capacitación es un método a través del cual se logra mejorar las actitudes, conductas y destrezas de los colaboradores para un buen desempeño en sus lugares de trabajo y lograr las metas de la organización.

De igual manera, Rengifo Maco (2018) señala que existen tres dimensiones señaladas por otros autores:

$\checkmark$ Primera dimensión: Necesidades de capacitación: responden a la insuficiencia o escasez de conocimientos necesarios, actitudes y habilidades que una persona debe adquirir para desempeñar adecuadamente las funciones correctas de su puesto (Guglielmetti).

$\checkmark$ Segunda dimensión: Plan de desarrollo de personas: es un escrito formal que sirve de puente móvil, entre lo que uno es hoy y lo que desea llegar a ser en el futuro. En consecuencia, hablamos de un "puente móvil" porque el plan debe ser flexible y adaptable, para que cada persona lo pueda ir modificando y afinando, conforme avance en su cumplimiento (Pimentel).

$\checkmark$ Tercera dimensión: Acciones de capacitación: las organizaciones favorecen las acciones de capacitación con enfoque de corto plazo, a fin de solucionar las dificultades que sitúan en peligro su capacidad (Martínez).

O’Regan et al. (2010) realizaron un estudio a más de 100 PYMES en Reino Unido encontrando que nunca se está demasiado preparado o experimentado. Siempre es importante recibir entrenamientos para mejorar y perfeccionar las habilidades y competencias.

\subsection{Sistemas adecuados de control (X6)}

Según el documento creado por Tesinaglobal (n.d.), un sistema de control es un tipo de sistema que se caracteriza por la presencia de una serie de elementos que permiten influir en el funcionamiento del sistema. La finalidad de un sistema de control es conseguir, mediante la manipulación de las variables de control, un dominio sobre las variables de salida, de modo que estas alcancen unos valores prefijados (consigna). 
Un sistema de control ideal debe ser capaz de conseguir su objetivo cumpliendo los siguientes requisitos:

$\checkmark$ Garantizar la estabilidad.

$\checkmark$ Ser tan eficiente como sea posible.

$\checkmark$ Ser fácilmente implementable y cómodo de operar.

Ahora bien, una teoría de los sistemas de control que es publicada por Ogata en 2003 menciona a los sistemas de control empresariales como aquel que está formado por muchos grupos. Cada tarea asignada a un grupo representará un elemento dinámico del sistema. Para la correcta operación de este sistema deben establecerse métodos de realimentación para informar de los logros de cada grupo.Un sistema empresarial es un sistema en lazo cerrado. Un buen diseño de este reducirá el control administrativo requerido. Obsérvese que las perturbaciones en este sistema son la falta de personal o de materiales, la interrupción de las comunicaciones, los errores humanos, etc.

Un estudio de Parra (2014) busca conocer las características que identifican los elementos y componentes del control interno en las mipymes y considera diferentes características como lo son el Ambiente de Control, la Evaluación de Riesgos, las Actividades de Control, la Información y Comunicación y el Monitoreo. El control interno es un instrumento de gran valor, aunque su utilización no garantiza que se conseguirán las metas propuestas. Las decisiones en las empresas las toman los directivos, y no dependen de la estructura de control interno.

\section{MÉTODO}

Esta investigación es cuantitativa ya que se utilizará una encuesta para medir y cuantificar la relación entre las variables. Además, Se utilizan los tipos de investigación Exploratoria, Descriptiva, Correlacional y Explicativa. El diseño de la investigación es no experimental ya que no se manipulan las variables del estudio. Es transeccional ya que se analiza la situación en un momento especifico y las técnicas que se usaron para la investigación fueron la documental, bibliográfica y de campo, usando la encuesta. La cual es de carácter cuantitativo puesto que miden y cuantifican las respuestas, consiste en 45 preguntas en total divididas en 3 secciones y cubriendo tanto la variable dependiente como las independientes.

La primera sección, con 4 preguntas, cubre el perfil del encuestado con información como posición, nivel de estudio, años laborando, etc. siempre manteniendo las preguntas lejos de la información personal sensible. Después viene la segunda sección que cubre el perfil de la empresa, con 6 preguntas. Aquí se tocan puntos como año de fundación, ubicación geográfica, número de empleados, actividades, rango de ventas anuales, etc. para después pasar a la tercera sección en donde se mencionan tanto la única variable dependiente como las 6 variables independientes, aquí hay 35 preguntas en total, 5 por cada variable. Se utiliza la escala Likert para obtener los resultados, donde 1 = Totalmente en desacuerdo, $2=$ En desacuerdo, $3=$ ni en desacuerdo ni de acuerdo, 4 = De acuerdo y $5=$ Totalmente de acuerdo. 
La población de estudio son las empresas medianas manufactureras de la fabricación de productos metálicos y de plástico sin reforzamiento y el sujeto de estudio son los dueños o directivos. Solamente se seleccionaron a las empresas localizadas en el Municipio de Apodaca en el Estado de Nuevo León, que según el Directorio Estadístico Nacional de Unidades Económicas (DENUE), existen 24 entidades como estas. Se utilizó la fórmula estadística, tomando en cuenta una confianza del 95\% y el resultado de la muestra fueron 20 empresas. Para analizar los resultados, se utilizará el método de análisis estadístico de regresión lineal múltiple.

Lo que se realizó para obtener los resultados de la encuesta fue por medio digital ya que las condiciones de salud no permitieron realizar solicitudes ni seguimiento presencial de las encuestas. Se creó un link que llevaría directamente al portal en donde el encuestado podía ver y contestar las diferentes preguntas para que a su vez, estas fueran enviadas de regreso para su análisis en un software estadístico para poder visualizar las respuestas y sus correlaciones.

\section{RESULTADOS}

En la encuesta se realizaron 4 preguntas muy sencillas que nos ayudan a poder definir el perfil del encuestado de una manera rápida y directa. Primero que nada, se identifica la posición de la persona en la compañía ya que es distinto la manera en que un dueño y un empleado ven las estrategias. Después se busca catalogar a las personas por su antigüedad en la compañía y el número de personas a su cargo, esto debido a que se supondría que mientras más tiempo se esté en la empresa se tiende a estar de acuerdo y en sintonía con la cultura organizacional y las metas de esta. Además, aunque no necesariamente, una persona con más tiempo en la compañía tendría más posibilidades de tener gente a su cargo. Finalmente se busca tener información acerca del nivel de estudio para ver si hay relación entre estudios y posición laboral. Los resultados fueron interesantes:

- Hubo una cantidad de respuesta de propietarios similar a la cantidad de respuestas de empleados sin cargo gerencial lo cual abre posibilidades para que las respuestas sean variadas.

- Respecto a la antigüedad laboral, la mayoría de las personas cuentan con una antigüedad de menos de 5 años, esto podría deberse a que las empresas son recientes o que simplemente lleva poco tiempo en esa compañía.

- La gran mayoría de personas no tienen personal a cargo y esto puede hacer que las decisiones que toman sean pensadas más con la visión individual que de equipo.

- En los niveles de estudio, la aplastante mayoría cuenta con nivel de estudio superior, se puede deber a la zona geográfica que goza Nuevo León.

\section{Graficas 1.- Resultados del Perfil del Encuestado}




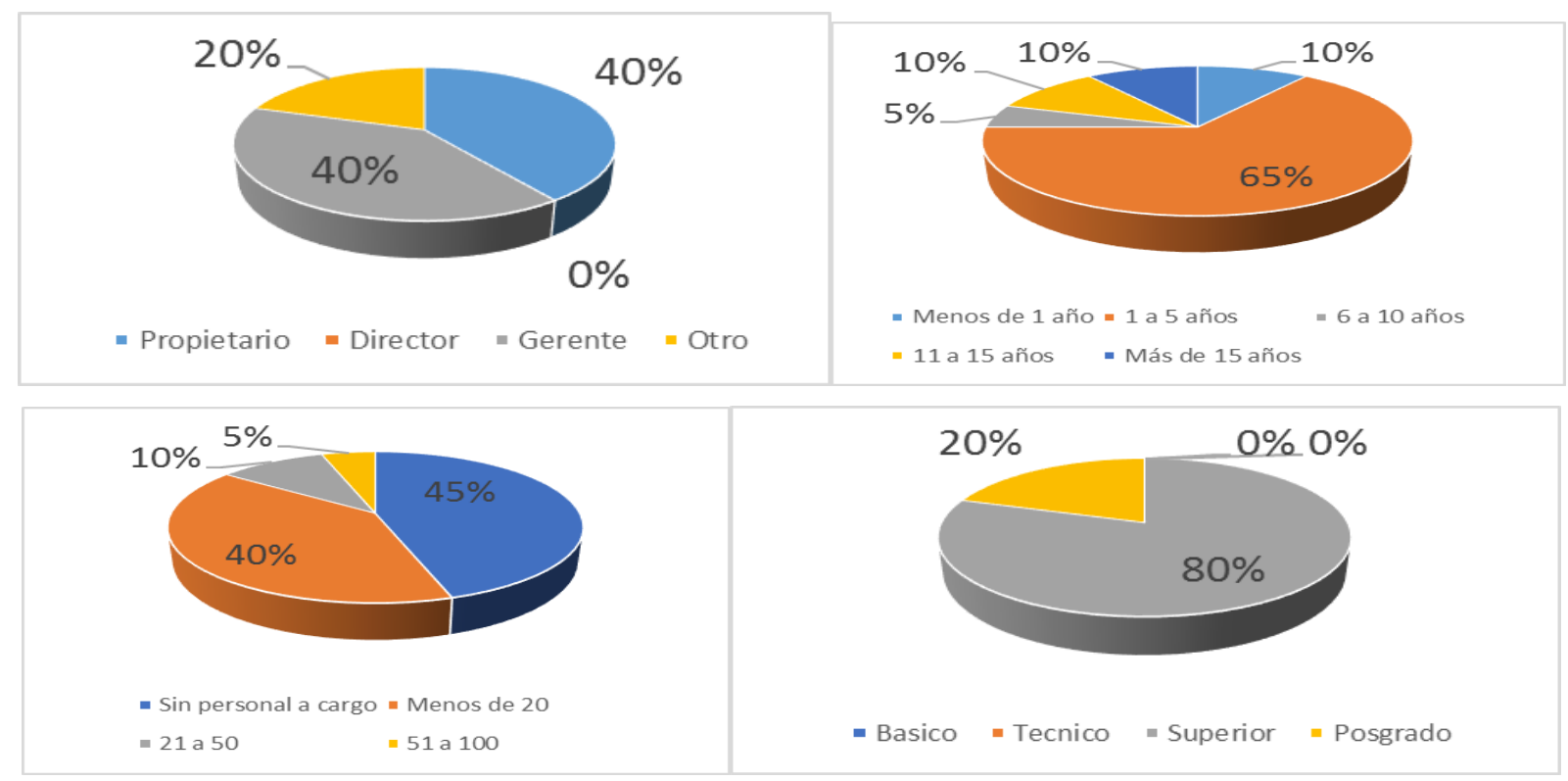

Fuente: Elaboración propia a partir de los resultados

Ahora, respecto al perfil de la empresa también se ubicaron preguntas con la finalidad de hacer perfil de las empresas y reafirmar que pertenecen al grupo de estudio. Año de fundación, número de empleados, ventas anuales y porcentaje de crecimiento es la información que tenemos para identificar el perfil. Por lo que se presentan los resultados:

- La antigüedad de la empresa nos dice muchísimo, si es más antigua probablemente haya podido ir perfeccionando sus operaciones, si es más reciente tal vez tendrá nuevas tácticas para acatar el mercado. En general, hubo un equilibrio.

- El número de empleados es bastante importante ya que podría estar ligado a la antigüedad o bien a que la empresa no haya crecido tanto si fuera muy antigua y mantuviera una plantilla pequeña.

- Las ventas son importantísimas para ubicar si hay competitividad de fondo o no. A menos que sean compañías muy recientes.

- Finalmente, saber si las empresas estudiadas han tenido mejoras respecto a años pasados. Si han vendido más, es buena señal, si no, tal vez haya cosas por mejorar.

Graficas 1.- Resultados del Perfil de la Empresa 


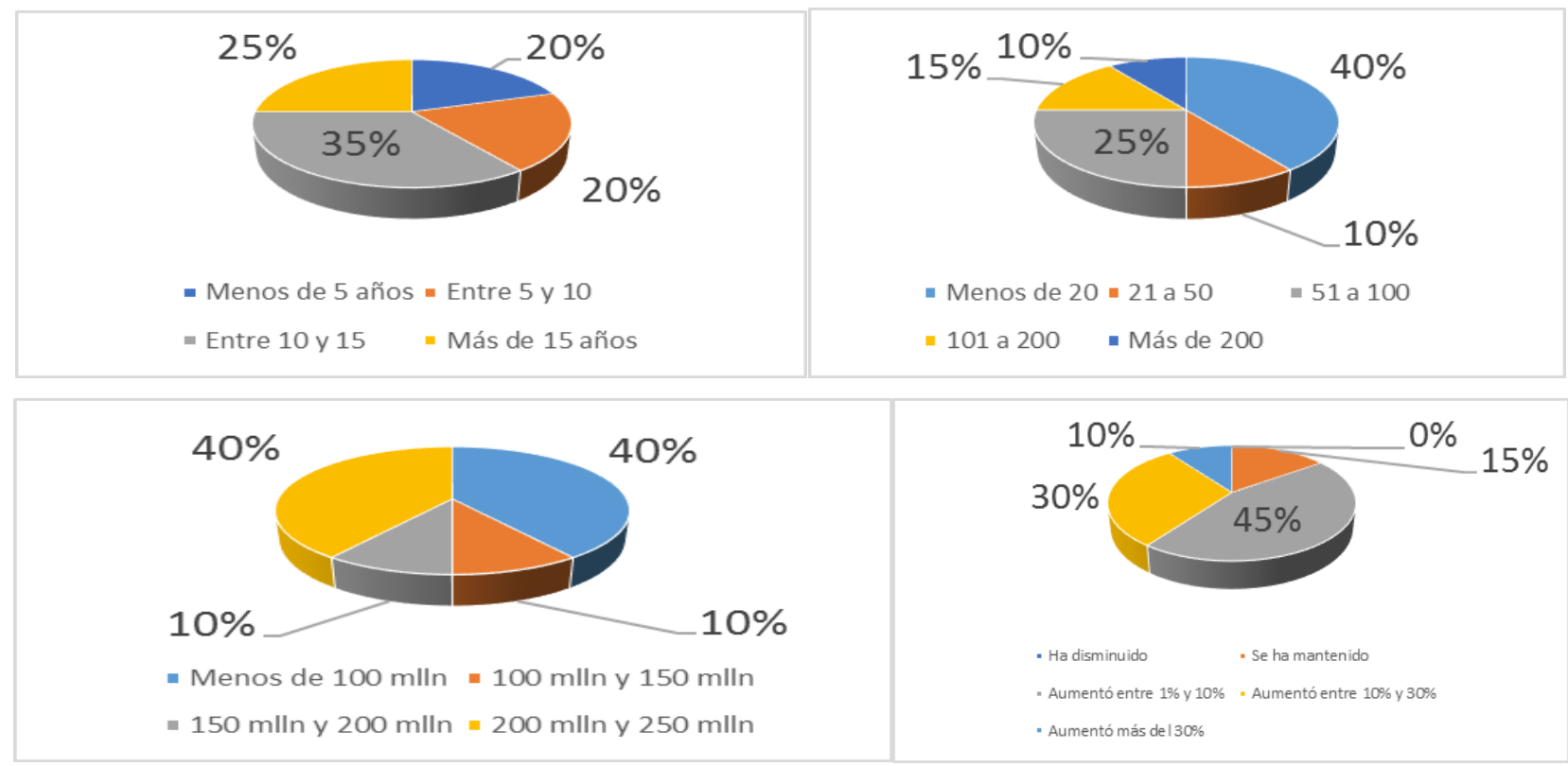

Fuente: Elaboración propia a partir de los resultados

\subsection{Comprobación de hipótesis}

Para este estudio se utilizó un análisis de regresión lineal múltiple puesto que se evalúan múltiples variables independientes como contar con recursos tecnológicos, innovación en productos, tener finanzas sanas, contar con un plan de negocios, capacitación del personal y tener sistemas adecuados de control con respecto a una dependiente que es indiscutiblemente la Competitividad.

Se utilizó el software estadístico SPSS para poder obtener estos cálculos matemáticos con mayor confianza y aminorar cualquier posible error aunque en una revisión simple de los resultados podemos encontrar que las respuestas son variadas y la opinión se encuentra dividida en algunos casos.

Tabla 1. Resultados de las Hipótesis

\begin{tabular}{|c|c|c|}
\hline VARIABLES & SIG & RESULTADOS \\
\hline X1 Recursos Tecnológicos & 0.740 & RECHAZADA \\
\hline X2 Innovación & 0.015 & ACEPTADA \\
\hline X3 Fianzas Sanas & 0.852 & RECHAZADA \\
\hline X4 Plan de Negocio & 0.722 & RECHAZADA \\
\hline X5 Capacitación & 0.049 & ACEPTADA \\
\hline X4 Sistemas de Control & 0.057 & RECHAZADA \\
\hline
\end{tabular}

Fuente: Elaboración propia a partir de los resultados 


\section{CONCLUSIONES}

Para concluir, podríamos determinar que a lo largo del documento se analizaron los antecedentes de la importancia de las PYMES a nivel, falta de competitividad en las PYMES de Nuevo León y el impacto que esto puede llegar a tener, así como cuales serían las posibles soluciones a ello. Así mismo se hizo una extensa revisión teórica a diferentes documentos de distintos autores para dar sustento a las variables analizadas. Se revisaron teorías como la que concierne a los recursos tecnológicos en el campo educativo y laboral, la teoría del desenvolvimiento económico que plantea la idea de la innovación como parte endógena del sistema económico, la teoría de planeación financiera en PYMES de México sugerido por Masilo y Aguirre en 2017, la teoría de Porter que habla de los determinantes de la ventaja competitiva, entre otras.

Para lograr tener competitividad siendo una empresa mediana, es necesario contar con diferentes características y/o habilidades como lo son los recursos tecnológicos, tener finanzas sanas, capacitar al personal, entre otras. Podemos concluir, de manera general, en que estos factores sí influyen directamente con la competitividad de las empresas ya que en una encuesta realizada a personas activamente trabajando en empresas medianas se obtuvieron respuestas que indicaban que sí se deben de cumplir con dichas variables para lograr tener competitividad.

Asimismo, yo recomendaría no considerar este o ningún otro estudio acerca de la competitividad como un resultado o dictamen final porque la competencia tiende a irse renovando y actualizando de manera regular por lo que en un futuro las características aquí mencionadas podrían no ser las más importantes al igual que otros modelos de competencia podrían crearse y desarrollarse para poder tener más impacto en el mercado. La investigación y los estudios deben de continuar, deben de confrontarse y seguirse actualizando. En un futuro tal vez la competitividad dependa de otros factores o alguno de los ya mencionados cobre importancia superior por lo que habrá de enfocarse en lo que ofrezca mayores beneficios. 


\section{REFERENCIAS}

Ahmedova, S. (2015). Factors for increasing the competitiveness of small and medium-sized enterprises (SMEs) in Bulgaria. Procedia-Social and Behavioral Sciences, 195, 1104-1112. Encontrado en la base de datos de Google Scholar

Canal, Lukas. (n.d.). La evolución de las PYMES en la era de la digitalización. Encontrado en https://franquiciasdemexico.org.mx/la-evolucion-las-PYMES-en-la-era-la-digitalizacion/

Cuevas Vargas, H. (2016). La influencia de la innovación y la tecnología en la competitividad de las PYMES manufactureras del estado de Aguascalientes. Encontrado en la base de datos de Google Scholar.

DENUE. (2021). Directorio estadístico nacional de unidades económicas. Encontrado en https://www.inegi.org.mx/app/mapa/denue/default.aspx

Duran, Paloman. (2017). Microempresas, PYMES y objetivos de desarrollo sostenible. Encontrado en https://www.onu.org.mx/microempresas-PYMES-y-objetivos-de-desarrollo-

sostenible/\#: :text=Las\%20micro\%2C\%20peque\%C3\%B1as\%20y\%20medianas,empresarial\%20en\% 20todo\%20el\%20mundo.\&text=Las\%20estad\%C3\%ADsticas\%20de1\%20Banco\%20Mundial,PIB)\%2 0en\%20las\%20econom\%C3\%ADas\%20emergentes.

Económica, Gaceta. (2019). Cuatro entidades federativas aportaron el 40\% del PIB nominal nacional en 2018. Encontrado en https://www.gob.mx/shcp\%7Cgacetaeconomica/articulos/cuatro-entidades-federativasaportaron-el-40-del-pib-nominal-nacional-en-2018

García, M. L. S., \& Sánchez, B. T. (n.d.) Las mejores prácticas en la Mipyme Industrial Mexicana. Encontrado en http://congreso.investiga.fca.unam.mx/docs/xvi/docs/1W.pdf

González, J. G., \& De La Parra, J. P. N. (2017). Una visión de la innovación como elemento clave para mejorar la competitividad en las PYMES mexicanas. Red Internacional de Investigadores en Competitividad, 5(1). Encontrado en https://riico.net/index.php/riico/article/viewFile/575/779.

Ibarra Cisneros, M. A., González Torres, L. A., \& Demuner Flores, M. D. R. (2017). Competitividad empresarial de las pequeñas y medianas empresas manufactureras de Baja California. Estudios fronterizos, 18(35), 107-130. Encontrado en http://www.scielo.org.mx/scielo.php?pid=S018769612017000100107\&script=sci_arttext

Jiménez, R. A., Campos, P. C., \& Vargas-Hernández, J. G. (2020). Los factores internos de las Pymes y su influencia en la competitividad (Caso sector comercio en Lázaro Cárdenas Michoacán). Horizontes Empresariales, 19(2), 28-45. Encontrado en http://www.adpt.ubiobio.cl/index.php/HHEE/article/view/3907/3654

Labarca, N. (2007). Consideraciones teóricas de la competitividad empresarial. Omnia, 13(2), 158-184. Encontrado en https://www.redalyc.org/pdf/737/73713208.pdf 
Masilo, A. E., \& Aguirre, M. G. (2017). Estudio de revisión sobre la planeación financiera y propuesta de modelo empírico para PYMES de México. Cimexus, 11(2), 73-106. Encontrado en la base de datos de Google Scholar.

Neuman, Jacobo. (2020). ¿Cómo operan las PYMES, incluyendo la tuya, en México o en el mundo? ¿Quieres saber cuáles son tus calificaciones para poder mejorarlas y qué hacer al respecto? Encontrado en https://www.pulsopyme.com/como-operan-en-la-actualidad-las-PYMES-en-mexico/

O'Regan, N., Stainer, L., \& Sims, M. (2010). Training in SMEs and its relationship to profitability. International journal of human resources development and management, 10(2), 166-181. Encontrado en https://uhra.herts.ac.uk/bitstream/handle/2299/5762/905346? sequence=1

Parra, L. I. C. (2014). Los sistemas de control interno en las Mipymes y su impacto en la efectividad empresarial. Revista En-contexto/ISSN: 2346-3279, (2), 129-146. Encontrado en la base de datos de Google Scholar.

Pavón, L. (2016). Inclusión financiera de las PYMES en el Ecuador y México. Encontrado en https://repositorio.cepal.org/bitstream/handle/11362/40848/S1601083_es.pdf?sequence=1

Pinto, J., \& Rodríguez-Moreno, J. (2018). Medición del impacto de la innovación medio ambiental sobre el empleo en Ecuador 2012-2014. X-pedientes Económicos, 2(4), 37-61. Encontrado en la base de datos de Google Scholar.

Prasanna, R. P. I. R., Jayasundara, J. M. S. B., Naradda Gamage, S. K., Ekanayake, E. M. S., Rajapakshe, P. S. K., \& Abeyrathne, G. A. K. N. J. (2019). Sustainability of smes in the competition: A systemic review on technological challenges and sme performance. Journal of Open Innovation: Technology, Market, and Complexity, 5(4), 100. Encontrado en la base de datos de Google Scholar.

Rengifo Maco, R. M. (2018). Proceso de capacitación y el desempeño laboral del personal administrativo del organismo de evaluación y fiscalización ambiental, Lima 2017. Encontrado en https://repositorio.ucv.edu.pe/bitstream/handle/20.500.12692/16316/Rengifo_MRM.pdf?sequence=1

Saavedra-García, M. L., Tapia-Sánchez, B., \& de los Ángeles Aguilar-Anaya, M. (2016). La gestión financiera en las PYMES del Distrito Federal, México. Revista Perspectiva Empresarial, 3(2), 55-69. Encontrado en la base de datos de Google Scholar.

SAP Blogs. (2013). La importancia global de las PYMES. Encontrado en https://news.sap.com/latinamerica/2013/10/importancia-global-

PYMES/\#: :text=Las\%20peque\%C3\%B1as\%20y\%20medianas\%20empresas,las\%20exportaciones\% 20y\%20al\%20comercio.\&text=Exportan\%20productos\%20y\%20servicios\%20para\%20nichos\%20de $\% 20$ mercado\%20m\%C3\%A1s\%20especializados.

Singh, R. K., Garg, S. K., \& Deshmukh, S. G. (2010). The competitiveness of SMEs in a globalized economy. Management research review, 33(1), 54. Encontrado en: https://www.researchgate.net/profile/Rajesh_Singh63/publication/235270243_The_competitiveness_o f_SMEs_in_a_globalized_economy_Observations_from_China_and_India/links/5548a9720cf2e2031b 
38a91e/The-competitiveness-of-SMEs-in-a-globalized-economy-Observations-from-China-andIndia.pdf

$\begin{array}{lllll}\text { Tesinaglobal } & \text { (n.d.). } & \text { Sistemas entrol. } & \text { Encontrado } & \text { en }\end{array}$ https://upcommons.upc.edu/bitstream/handle/2099.1/3330/34059-5.pdf?sequence=5

Valverde Arana, G. J. (2011). Plan de negocios para la creación de un centro de asesoramiento para la competitividad empresarial (cace) dirigida a las pequeñas y medianas empresas de la ciudad de Babahoyo (Bachelor's thesis, Babahoyo: UTB. 2011). Encontrado en http://dspace.utb.edu.ec/bitstream/handle/49000/53/T-UTB-FAFI-IC-

000002.pdf?sequence $=6 \&$ isAllowed $=y$

Vargas Urzola, A. (2002). Modelo para la elaboración de un plan de negocios para las empresas pequeñas y medianas. Estudios Gerenciales, 18(82), 93-108. Encontrado en http://www.scielo.org.co/scielo.php?pid=S0123-59232002000100005\&script=sci_arttext\&tlng=en

Ycaza, D. E. M., \& Sánchez-Riofrío, A. M. (2016). Factores de competitividad orientados a la pequeña y mediana empresa (PYME) en Latinoamérica: revisión de la literatura. Revista San Gregorio, 2(15), 104111.

Encontrado

en

http://revista.sangregorio.edu.ec/index.php/REVISTASANGREGORIO/article/viewFile/275/9 